\title{
Short history of Héctor Rubinstein's scientific career and his years in Sweden
}

Lars Bergström, Jan Conrad, Joakim Edsjö, Ariel Goobar, Per Olof Hulth, Edvard Mörtsell

Oskar Klein Centre

Department of Physics

Stockholm University

S-106 91 Stockholm, Sweden

E-mail: Ibelfysik.su.se, conradefysik.su.se, edsjolfysik.su.se,

arielefysik.su.se, hulthefysik.su.se, edvardefysik.su.se

\section{Ulf Danielsson}

Department of Physics and Astronomy

Uppsala University

S-75108 Uppsala, Sweden

E-mail: ulf.danielssonephysics.uu.se

Quarks, Strings and the Cosmos - Héctor Rubinstein Memorial Symposium,

AlbaNova, Stockholm, Sweden

August 9-11, 2010 
At the time of his death, Hector Rubinstein was professor emeritus at Uppsala University, and guest researcher and doctor honoris causa at Stockholm University. Hector was born in Argentina in 1933 and left the country in the fifties to study physics in the USA. After completing his $\mathrm{PhD}$ at Columbia University he moved as a postdoctoral fellow to Paris. In an early work he studied the mathematical theory for strong interaction, SU(3), which had shortly before been developed by the Nobel laureate Murray Gell-Mann, and which later became known as the quark model. In the beginning of the 80's, during a stay at the Rutherford laboratory in England, he made a very valuable contribution to Physics working with at that time new field-theoretical methods, i.e. sum rules that could be used to calculate particles masses and interactions in QCD. In 1985, he wrote a highly cited review on this subject together with L.J. Reinders and S. Yazaki.

After his stay in Paris, he became faculty at the Weizmann institute, where he attracted a number of excellent students, among them Gabriele Veneziano, Florian Scheck and Miguel Virasoro. The measured correlations in the strong force lead to the Veneziano model (or dual resonance model) which later was reinterpreted by Holger Bech-Nielsen and the 2008 Nobel-prize winner, Yoichiro Nambu, as a model for "strings". In a way, string theory was born in his research group at Weizmann.

Starting in the early 70's Hector sustained scientific contacts to Scandinavia (his wife being Swedish), resulting in his emigration to Sweden in 1984 and his appointment as professor at Uppsala University. Owing to his scientific reputation and his personal charisma he became very influential in developing the fields of particle astrophysics and string theory in Sweden. Hector, never shying away from controversy and pointing out incongruities also made important contributions to the public discurs of Physics research in Sweden.

In later years, Hector made a significant mark on the scientific publication process. Hector early realized that the internet would eventually revolutionize the way physicists publish their results. He therefore founded the Journal of High Energy Physics in 1997, the first in a series of J-journals which under Hectors guidance became well established with an excellent reputation. This is most recently illustrated by the publication of complete scientific documentation of the LHC machine and detectors in the Journal of Instrumentation.

Apart from particle astrophysics, his later work focused on cosmology, in particular the role of magnetic fields in the Early Universe. His last paper, written only weeks before his death, addressed whether primordial magnetic fields could be produced in inflation. Hector was very active also after his formal retirement in the year 2000 and except for his own research and his work with the journals he became a well respected mentor always willing to give his advice to young researchers and graduate students.

Hector had a great sense of humour and broad interests, and with his seemingly infinite stock of anecdotes, discussing with him was not only worthwhile but also incredibly fun.

He will be sorely missed. 